\title{
HOLOMORPHIC CONTRACTIBILITY OF TEICHMÜLLER SPACES
}

\author{
SAMUEL L. KRUSHKAL
}

\begin{abstract}
The problem of holomorphic contractibilty of Teichmüller spaces $\mathbf{T}(0, n)$ of the punctures spheres $(n>4)$ arose in the 1970s in connection with solving the algebraic equations in Banach algebras. We provide a positive solution of this problem.
\end{abstract}

2010 Mathematics Subject Classification: Primary: 30C55, 30F60; Secondary: 30F35, $46 \mathrm{G} 20$

Key words and phrases: Teichmüller spaces, Fuchsian group, quasiconformal deformations, holomorphic contractibility, univalent function, Schwarzian derivative, holomorphic sections

\section{INTRODUCTORY REMARKS AND STATEMENT OF RESULTS}

Let $X$ be a complex Banach manifold which is contractible to its point $x_{0}$, that is, there exists a continuous map $F: X \times[0,1] \rightarrow X$ with $F(x, 0)=x$ and $F(x, 1)=x_{0}$ for all $x \in X$. If the map $F$ can be chosen so that for every $t \in[0,1]$ the map $F_{t}: x \mapsto F(x, t)$ of $X$ to itself is holomorphic and $F_{t}\left(x_{0}\right)=x_{0}$, then $X$ is called holomorphically contractible to $x_{0}$.

The holomorphic contractibility of the finite dimensional Stein manifolds closely relates to the classical Oka-Grauert $h$-principle.

First notrivial example of a (unbounded) contractible domain in $\mathbb{C}^{2}$, which does not be holomorphically contractible, was provided by Hirchkowitz [10]. Zaidenberg and Lin [21], 22] (see also [20]) have established that there exist contractible bounded domains of holomorphy in $\mathbb{C}^{n}, n>1$, that are not holomorphically contractible. All these domains are the polynomial polyhedrons .

This fact became underlying for the problem of holomorphic contractibility of Teichmüller spaces $\mathbf{T}(0, n)$ of the spheres with $n>4$ punctures (the case $n=4$ is trivial, since $\mathbf{T}(0,4)$ is conformally equivalent to unit disk).

This problem arose many years ago in connection with solving algebraic equations in commutative Banach algebras and goes back to Gorin (see, e.g., 9]). It still remains open for any Teichmüller space of dimension greater than 1 (which are topologically contractible).

The simplest example of holomorphically contractible domains in complex Banach spaces is given by starlike domains. However all Teichmüller spaces of sufficiently great dimensions are not stalike (see [13, [14, [19]).

Earle [6] established the holomorphic contractibility of two modified Teichmüller spaces related to asymptotically conformal maps.

We show that the solution of this problem is positive:

Theorem. Any space $\mathbf{T}(0, n)$ with $n>4$ is holomorphically contractible.

As a simple consequence of this theorem, one obtains the holomorphic contractibility of two Teichmüller spaces of Riemann surfaces $X$ of positive genus, because the space $\mathbf{T}(0,5)$ is biholomorphically equivalent to the space $\mathbf{T}(1,2)$ of twice punctured tori and $\mathbf{T}(0,6)$ is equivalent to the

Date: July 18, 2018 (contractinterpol.tex). 
space $\mathbf{T}(2,0)$ of closed Riemann surfaces of genus 2 (see, e.g., [3]). We present this fact in the following

Corollary. The spaces $\mathbf{T}(1,2)$ and $\mathbf{T}(2,0)$ are holomorphically contractible.

Note that the proof of the theorem involves certain specific features of spaces $\mathbf{T}(0, n)$ and that the isomorphisms $\mathbf{T}(1,2) \simeq \mathbf{T}(0,5)$ and $\mathbf{T}(2,0) \simeq \mathbf{T}(0,6)$ are exceptional.

\section{A GLIMPSE OF TEICHMÜLLER SPACES}

We briefly recall some needed facts from the Teichmüller space theory.

2.1. Consider the ordered $n$-tuples of points

$$
\mathbf{a}=\left(0,1, a_{1}, \ldots, a_{n-3}, \infty\right), \quad n>4,
$$

with distinct $a_{j} \in \mathbb{C} \backslash\{0,1\}$ and the corresponding punctured spheres

$$
X_{\mathbf{a}}=\widehat{\mathbb{C}} \backslash\left\{0,1, a_{1} \ldots, a_{n-3}, \infty\right\}, \quad \widehat{\mathbb{C}}=\mathbb{C} \cup\{\infty\},
$$

regarded as the Riemann surfaces of genus zero. Fix a collection $\mathbf{a}^{0}=\left(0,1, a_{1}^{0}, \ldots, a_{n-3}^{0}, \infty\right)$ with $1<a_{1}^{0}<\cdots<a_{n}^{0}<\infty$ defining the base point $X_{\mathbf{a}^{0}}$ of Teichmüller space $\mathbf{T}(0, n)=\mathbf{T}\left(X_{\mathbf{a}^{0}}\right)$. Its points are the equivalence classes $[\mu]$ of Beltrami coefficients from the ball

$$
\operatorname{Belt}(\mathbb{C})_{1}=\left\{\mu \in L_{\infty}(\mathbb{C}):\|\mu\|_{\infty}<1\right\},
$$

under the relation that $\mu_{1} \sim \mu_{2}$ if the corresponding quasiconformal homeomorphisms $w^{\mu_{1}}, w^{\mu_{2}}$ : $X_{\mathbf{a}^{0}} \rightarrow X_{\mathbf{a}}$ (the solutions of the Beltrami equation $\bar{\partial} w=\mu \partial w$ with $\mu=\mu_{1}, \mu_{2}$ ) are homotopic on $X_{\mathbf{a}^{0}}$ (and hence coincide in the points $\left.0,1, a_{1}^{0}, \ldots, a_{n-3}^{0}, \infty\right)$. This models $\mathbf{T}(0, n)$ as the quotient space

$$
\mathbf{T}(0, n)=\operatorname{Belt}(\mathbb{C})_{1} / \sim
$$

with complex Banach structure of dimension $n-3$ inherited from the ball Belt $(\mathbb{C})_{1}$. Note that $\mathbf{T}(0, n)$ is a complete metric space with intrinsic Teichmüller metric defined by quasiconformal maps. By Royden's theorem, this metric equals the Kobayashi metric determined by the complex structure.

Another canonical model of $\mathbf{T}(0, n)=\mathbf{T}\left(X_{\mathbf{a}^{0}}\right)$ is obtained using the uniformization of Riemann surfaces and the holomorphic Bers embedding of Teichmüller spaces. Consider the upper and lower half-planes

and the ball

$$
U=\{z=x+i y: y>0\}, \quad U^{*}=\{z \in \widehat{\mathbb{C}}: y<0\}
$$

$$
\operatorname{Belt}(U)_{1}=\left\{\mu \in L_{\infty}(\mathbb{C}): \mu \mid U^{*}=0,\|\mu\|_{\infty}<1\right\},
$$

and call the Beltrami coefficients $\mu_{1}$ and $\mu_{2}$ from this ball equivalent if $w^{\mu_{1}}=w^{\mu_{2}}$ on the real axes $\mathbb{R}=\partial U^{*}$ (hence on $\overline{U^{*}}$ ). Such equivalence classes $[\mu]$ are the points of the universal Teichmüller space $T$ and correspond one-to-one to the Schwarzian derivatives

$$
S_{w}(z)=\frac{w^{\prime \prime \prime}(z)}{w^{\prime}(z)}-\frac{3}{2}\left(\frac{w^{\prime \prime}(z)}{w^{\prime}(z)}\right)^{2}
$$

of maps $w=w^{\mu}$ in $U^{*}$. These derivatives form a bounded domain in the complex Banach space $\mathbf{B}=\mathbf{B}\left(U^{*}\right)$ of hyperbolically bounded holomorphic functions $\varphi$ in the lower half plane with norm

$$
\|\varphi\|=\sup _{\Delta^{*}} 4 y^{2}|\varphi(z)| .
$$

This domain is contained in the ball $\left\{\|\varphi\|_{\mathbf{B}}<6\right\}$. 
The map $\phi: \mu \rightarrow S_{w^{\mu}}$ is holomorphic and descends to a biholomorphic map of the space $\mathbf{T}$ onto this domain, which we will identify with $\mathbf{T}$. It contains as complex submanifolds the Teichmüller spaces of all hyperbolic Riemann surfaces and of Fuchsian groups.

2.2. Using the holomorphic universal covering map $h: U \rightarrow X_{\mathbf{a}^{0}}$, one represents the surface $X_{\mathbf{a}^{0}}$ as the quotient space $U / \Gamma_{0}$ (up to conformal equivalence), where $\Gamma_{0}$ is a torsion free Fuchsian group of the first kind acting discontinuously on $U \cup U^{*}$. The functions $\mu \in L_{\infty}\left(X_{\mathbf{a}^{0}}\right)$ are lifted to $U$ as the Beltrami $(-1,1)$-measurable forms $\widetilde{\mu} d \bar{z} / d z$ in $U$ with respect to $\Gamma_{0}$ which satisfy $(\widetilde{\mu} \circ \gamma) \overline{\gamma^{\prime}} / \gamma^{\prime}=\widetilde{\mu}, \gamma \in \Gamma_{0}$ and form the Banach space $L_{\infty}\left(U, \Gamma_{0}\right)$.

We extend these $\widetilde{\mu}$ by zero to $U^{*}$ and consider the unit ball $\operatorname{Belt}\left(U, \Gamma_{0}\right)_{1}$ of $L_{\infty}\left(U, \Gamma_{0}\right)$. Then the corresponding Schwarzians $S_{w^{\widetilde{\mu}} \mid U^{*}}$ belong to the universal Teichmüller space $\mathbf{T}$ and the subspace of such Schwarzians is regarded as the Teichmüller space $\mathbf{T}\left(\Gamma_{0}\right)$ of the group $\Gamma_{0}$. It is canonically isomorphic to the space $\mathbf{T}\left(X_{\mathbf{a}^{0}}\right)$. Moreover,

$$
\mathbf{T}\left(\Gamma_{0}\right)=\mathbf{T} \cap \mathbf{B}\left(\Gamma_{0}\right),
$$

where $\mathbf{B}\left(\Gamma_{0}\right)$ is a $(n-3)$-dimensional subspace of $\mathbf{B}$ which consists of elements $\varphi \in \mathbf{B}$ satisfying

$$
(\varphi \circ \gamma)\left(\gamma^{\prime}\right)^{2}=\varphi \text { for all } \gamma \in \Gamma_{0}
$$

(holomorphic $\Gamma_{0}$-automorphic forms of degree -4 ); see, e.g. [16]. This leads to the representation of the space $\mathbf{T}\left(X_{\mathbf{a}^{0}}\right)$ as a bounded domain in the complex Euclidean space $\mathbb{C}^{n-3}$.

Note also that the space $\mathbf{B}$ is dual to the subspace $A_{1}\left(U^{*}\right)$ in $L_{1}\left(U^{*}\right)$ formed by integrable holomorphic functions in $U^{*}$, while $B\left(\Gamma_{0}\right)$ has the same elements as the space $A_{1}\left(U^{*}, \Gamma_{0}\right)$ of integrable holomorphic forms of degree -4 with norm $\|\varphi\|=\iint_{U^{*} / \Gamma_{0}}|\varphi(z)| d x d y$.

\section{PROOF OF THEOREM}

$\mathbf{1}^{0}$. We precede the proof of the theorem with several lemmas which follow [15].

First observe that collections (1) fill a domain $\mathcal{D}_{n}$ in $\mathbb{C}^{n-3}$ obtained by deleting from this space the hyperplanes $\left\{z=\left(z_{1}, \ldots, z_{n-3}\right): z_{j}=z_{l}, j \neq l\right.$, and with $z_{1}=0, z_{2}=1$. This domain represents the Torelli space of the spheres $X_{\mathbf{a}}$ and is covered by $\mathbf{T}(0, n)$. Namely, we have (cf. e.g., [11]; [18], Section 2.8)

Lemma 1. The holomorphic universal covering space of $\mathcal{D}_{n}$ is the Teichmüller space $\mathbf{T}(0, n)$. This means that for each punctured sphere $X_{\mathbf{a}}$ there is a holomorphic universal covering

$$
\pi_{\mathbf{a}}: \mathbf{T}(0, n)=\mathbf{T}\left(X_{\mathbf{a}}\right) \rightarrow \mathcal{D}_{n} .
$$

The covering map $\pi_{a}$ is well defined by

$$
\pi_{\mathbf{a}} \circ \phi_{\mathbf{a}}(\mu)=\left(0,1, w^{\mu}\left(a_{1}\right), \ldots, w^{\mu}\left(a_{n-3}\right), \infty\right),
$$

where $\phi_{\mathbf{a}}$ denotes the canonical projection of the ball $\operatorname{Belt}(U)_{1}$ onto the space $\mathbf{T}\left(X_{\mathbf{a}}\right)$.

This lemma yields also that the truncated collections $\mathbf{a}_{*}=\left(a_{1}, \ldots, a_{n-3}\right)$ provide the local complex coordinates on the space $\mathbf{T}(0, n)$ and define its complex structure.

These coordinates are simply connected with the Bers local complex coordinates on $\mathbf{T}(0, n)$ (related to basises of the tangent spaces to $\mathbf{T}(0, n)$ at its points, see [2]) via standard variation of quasiconformal maps of $X_{\mathbf{a}}=U / \Gamma_{\mathbf{a}}$ (see, e.g., [12])

$$
\begin{aligned}
w^{\mu}(z) & =z-\frac{z(z-1)}{\pi} \iint_{\mathbb{C}} \frac{\mu(\zeta)}{\zeta(\zeta-1)(\zeta-z)} d \xi d \eta+O\left(\|\mu\|_{\infty}^{2}\right) \\
& =z-\frac{z(z-1)}{\pi} \sum_{\gamma \in \Gamma_{\mathbf{a}}} \iint_{U / \Gamma_{\mathbf{a}}} \frac{\mu(\gamma \zeta)\left|\gamma^{\prime}(\zeta)\right|^{2}}{\gamma \zeta(\gamma \zeta-1)(\gamma \zeta-z)} d \xi d \eta+O\left(\|\mu\|_{\infty}^{2}\right) .
\end{aligned}
$$


Now consider the ball Belt $(U)_{1}$ and call its elements $\mu$ defining the same point of the universal Teichmüller space $\mathbf{T}$-equivalent. The corresponding homeomorphisms $w^{\mu}$ coincide on the unit circle.

We now define on this ball another equivalence relation, letting $\mu, \nu \in \operatorname{Belt}(U)_{1}$ be equivalent if $w^{\mu}\left(a_{j}^{0}\right)=w^{\nu}\left(a_{j}^{0}\right)$ for all $j$ and the homeomorphisms $w^{\mu}, w^{\nu}$ are homotopic on the punctured sphere $X_{\mathbf{a}^{0}}$. Let us call such $\mu$ and $\nu$ strongly n-equivalent.

Lemma 2. If the coefficients $\mu, \nu \in \operatorname{Belt}(U)_{1}$ are $\mathbf{T}$-equivalent, then they are also strongly $n$ equivalent.

The proof of this lemma is given in [8].

In view of Lemmas 1 and 2 , the above factorizations of the ball Belt $(U)_{1}$ generate (by descending to the equivalence classes) a holomorphic map $\chi$ of the underlying space $\mathbf{T}$ into $\mathbf{T}(0, n)=\mathbf{T}\left(X_{\mathbf{a}^{0}}\right)$.

This map is a split immersion, i.e., it has local holomorphic sections. In fact, we have much more:

Lemma 3. The map $\chi$ is surjective and has a global holomorphic section $s: \mathbf{T}\left(X_{\mathbf{a}^{\mathbf{0}}}\right) \rightarrow \mathbf{T}$.

Proof. The surjectivity of $\chi$ is a consequence of the following interpolation result from [4].

Lemma 4. Given two cyclically ordered collections of points $\left(z_{1}, \ldots, z_{m}\right)$ and $\left(\zeta_{1}, \ldots, \zeta_{m}\right)$ on the unit circle $S^{1}=\{|z|=1\}$, there exists a holomorphic univalent function $f$ in the closure of the unit disk $\Delta=\{|z|<1\}$ such that $|f(z)|<1$ for $z \in \bar{\Delta}$ distinct from $z_{1}, \ldots, z_{m}$, and $f\left(z_{k}\right)=\zeta_{k}$ for all $k=1, \ldots, m$. Moreover, there exist univalent polynomials $f$ with such an interpolation property.

Since the interpolating function $f$ given by this lemma is regular up to the boundary, it can be extended quasiconformally across the boundary circle $S^{1}$ to the whole sphere $\widehat{\mathbb{C}}$. Hence, given a cyclically ordered collection $\left(z_{1}, \ldots, z_{m}\right)$ of points on $S^{1}$, then for any ordered collection $\left(\zeta_{1}, \ldots, \zeta_{m}\right)$ in $\widehat{\mathbb{C}}$, there is a quasiconformal homeomorphism $\widehat{f}$ of the whole sphere $\widehat{\mathbb{C}}$ carrying the points $z_{j}$ to $\zeta_{j}, j=1, \ldots, m$, and such that its restriction to the closed disk $\bar{\Delta}$ is biholomorphic on $\bar{\Delta}$.

Applying Lemma 1, one constructs quasiconformal extensions of $f$ lying in prescribed homotopy classes of homeomorphisms $X_{\mathbf{z}} \rightarrow X_{\mathbf{w}}$. The case of maps conformal in $U$ follows from above by conjugating the interpolating functions $f$ by the Möbius transformation $\zeta \mapsto i(1+\zeta) /(1-\zeta)$ mapping the disk $\Delta$ onto the lower half-plane.

To prove the assertion of Lemma 3 on holomorphic section for $\chi$, take a dense subset

$$
e=\left\{x_{1}, x_{2}, \ldots\right\} \subset X_{\mathbf{a}^{0}} \cap \mathbb{R}
$$

accumulating to all points of $\mathbb{R}$ and consider the surfaces

$$
X_{\mathbf{a}^{0}}^{m}=X_{\mathbf{a}^{0}} \backslash\left\{x_{1}, \ldots, x_{m}\right\}, \quad m \geq 1
$$

(having type $(0, n+m))$. The equivalence relations on $\operatorname{Belt}(\mathbb{C})_{1}$ for $X_{\mathbf{a}^{0}}^{m}$ and $X_{\mathbf{a}^{0}}$ generate a holomorphic map

$$
\chi_{m}: \mathbf{T}\left(X_{\mathbf{a}^{0}}^{m}\right) \rightarrow \mathbf{T}\left(X_{\mathbf{a}^{0}}\right) .
$$

Indeed, similar to Lemma 2, we have: if the coefficients $\mu, \nu \in \operatorname{Belt}(U)_{1}$ are strongly $(n+m)$ equivalent (i.e., homotopic on $X_{\mathbf{a}^{0}}^{m}$ ), then they are also strongly $n$-equivalent (homotopic on $X_{\mathbf{a}^{0}}$ ).

The needed homotopy on $X_{\mathbf{a}^{0}}$ is constructed in a standard way, for example, using the Ahlfors homotopy, letting $f(z, t)$ be the projection of the point on the noneuclidian segment between the corresponding covers of $f^{\mu}(z)$ and $f^{\nu}(z)$ on hyperbolic plane $U$ which divides this segment in the proportion $t:(1-t)$; this homotopy extends to omitting punctures $x_{j}$, together with $f^{\mu}$ and $f^{\nu}$ (cf. [1, [3]).

The inclusion map $j_{m}: X_{\mathbf{a}^{0}}^{m} \hookrightarrow X_{\mathbf{a}^{0}}$ forgetting the additional punctures generates a holomorphic embedding $s_{m}: \mathbf{T}\left(X_{\mathbf{a}^{0}}\right) \hookrightarrow \mathbf{T}\left(X_{\mathbf{a}^{0}}^{m}\right)$ inverting $\chi_{m}$. 
To present this section analytically, we uniformize the surface $X_{\mathbf{a}^{0}}^{m}$ by a torsion free Fuchsian group $\Gamma_{0}^{m}$ on $U \cup U^{*}$ so that $X_{\mathbf{a}^{0}}^{m}=U / \Gamma_{0}^{m}$. By (2), its Teichmüller space $\mathbf{T}\left(\Gamma_{0}^{m}\right)=\mathbf{T} \cap \mathbf{B}\left(\Gamma_{0}^{m}\right)$. It also can be regarded as a holomorphic universal cover of $\mathcal{D}_{n+m}$.

The holomorphic universal covering maps $h: U^{*} \rightarrow U^{*} / \Gamma_{0}$ and $h^{m}: U^{*} \rightarrow U^{*} / \Gamma_{0}^{m}$ are related by $j \circ h^{m}=h \circ \widehat{j}$, where $\widehat{j}$ is the lift of $j$. This induces a surjective homomorphism of the covering groups $\theta_{m}: \Gamma_{0}^{m} \rightarrow \Gamma_{0}$ by

$$
\widehat{j} \circ \gamma=\theta_{m}(\gamma) \circ \gamma, \quad \gamma \in \Gamma_{0}^{m},
$$

and the norm preserving isomorphism $\widehat{j}_{m, *}: \mathbf{B}\left(\Gamma_{0}\right) \rightarrow \mathbf{B}\left(\Gamma_{0}^{m}\right)$ by

$$
\widehat{j}_{m, *} \varphi=(\varphi \circ \widehat{j})\left(\hat{j}^{\prime}\right)^{2},
$$

which projects to the surfaces $X_{\mathbf{a}^{0}}$ and $X_{\mathbf{a}^{0}}^{m}$ as the inclusion of the space $Q\left(X_{\mathbf{a}^{0}}\right)$ of quadratic differentials corresponding to $\mathbf{B}\left(\Gamma_{0}\right)$ into the space $Q\left(X_{\mathbf{a}^{0}}^{m}\right)$ (cf. [7]). The equality (3) represents the section $s_{m}$ indicated above.

To investigate the limit function for $m \rightarrow \infty$, we embed $\mathbf{T}$ into the space $\mathbf{B}$ and compose each $s_{m}$ with a biholomorphism

$$
\eta_{m}: \mathbf{T}\left(X_{\mathbf{a}^{0}}^{m}\right) \rightarrow \mathbf{T}\left(\Gamma_{0}^{m}\right)=\mathbf{T} \cap \mathbf{B}\left(\Gamma_{0}^{m}\right) \quad(m=1,2, \ldots) .
$$

Then the elements of $\mathbf{T}\left(\Gamma_{0}^{m}\right)$ are represented in the form

$$
\widehat{s}_{m}(z, \cdot)=S_{f^{m}}\left(z ; X_{\mathbf{a}}\right),
$$

being parameterized by the points of $\mathbf{T}\left(X_{\mathbf{a}^{0}}\right)$.

Each $\Gamma_{0}^{m}$ is the covering group of the universal cover $h_{m}: U^{*} \rightarrow X_{\mathbf{a}_{0}^{m}}$, which can be normalized (conjugating appropriately $\Gamma_{0}^{m}$ ) by $h_{m}(-i)=-i, h_{m}^{\prime}(-i)>0$. Take its fundamental polygon $P_{m}$ obtained as the union of the circular $m$-gon in $\Delta^{*}$ centered at the infinite point with the zero angles at the vertices and its reflection with respect to one of the boundary arcs. These polygons increasingly exhaust the half-plane $U^{*}$ from inside; hence, by the Carathéodory kernel theorem, the maps $h_{m}$ converge to the identity map locally uniformly in $U^{*}$.

Since the set of punctures $e$ is dense on $\mathbb{R}$, it completely determines the equivalence classes $\left[w^{\mu}\right]$ and $S_{w^{\mu}}$ of $\mathbf{T}$, and the limit function $s(z, \cdot)=\lim _{m \rightarrow \infty} \widehat{s}_{m}(z, \cdot)$ maps $\mathbf{T}\left(X_{\mathbf{a}^{0}}\right)$ into $\mathbf{T}$, what canonically distinguishes a representative in each inverse image $\chi^{-1}\left(X_{\mathbf{a}}\right) \subset \mathbf{T}$.

For any fixed $X_{\mathbf{a}}$, this function is holomorphic on $U^{*}$; hence, by the well-known property of elements in the functional spaces with sup-norms, $s(z, \cdot)$ is holomorphic also in the norm of $\mathbf{B}$. This $s$ determines a holomorphic section of the original map $\chi$, which completes the proof of Lemma 3.

The holomorphy property indicated above is based on the following lemma of Earle [5].

Lemma 5. Let $E, T$ be open subsets of complex Banach spaces $X, Y$ and $B(E)$ be a Banach space of holomorphic functions on $E$ with sup norm. If $\varphi(x, t)$ is a bounded map $E \times T \rightarrow B(E)$ such that $t \mapsto \varphi(x, t)$ is holomorphic for each $x \in E$, then the map $\varphi$ is holomorphic.

Holomorphy of $\varphi(x, t)$ in $t$ for fixed $x$ implies the existence of complex directional derivatives

$$
\varphi_{t}^{\prime}(x, t)=\lim _{\zeta \rightarrow 0} \frac{\varphi(x, t+\zeta v)-\varphi(x, t)}{\zeta}=\frac{1}{2 \pi i} \int_{|\xi|=1} \frac{\varphi(x, t+\xi v)}{\xi^{2}} d \xi,
$$

while the boundedness of $\varphi$ in sup norm provides the uniform estimate

$$
\left\|\varphi(x, t+c \zeta v)-\varphi(x, t)-\varphi_{t}^{\prime}(x, t) c v\right\|_{B(E)} \leq M|c|^{2},
$$

for sufficiently small $|c|$ and $\|v\|_{Y}$. 
The image $s\left(\mathbf{T}\left(X_{\mathbf{a}^{0}}\right)\right)$ is an $(n-3)$-dimensional complex submanifold in $\mathbf{T}$ biholomorphically equivalent to $\mathbf{T}\left(\Gamma_{0}\right)$.

$\mathbf{2}^{0}$. We may now prove the theorem. Pick a collection $\mathbf{a}^{0}=\left(0,1, a_{1}^{0}, \ldots, a_{n-3}^{0}, \infty\right)$ and the marked surface $X_{\mathbf{a}^{0}}$ as indicated above, and consider its Teichmüller spaces $\mathbf{T}\left(X_{\mathbf{a}^{0}}\right)$ and $\mathbf{T}\left(\Gamma_{0}\right)$.

We embed the space $\mathbf{T}(0, n)=\mathbf{T}\left(X_{\mathbf{a}^{0}}\right)$ via $\mathbf{T}\left(\Gamma_{0}\right)$ in $\mathbf{T}$ and define on the space $\mathbf{T}\left(\Gamma_{0}\right)$ a holomorphic homotopy using the maps

$$
W^{\mu}=\sigma^{-1} \circ w^{\mu} \circ \sigma, \quad \mu \in \operatorname{Belt}(U)_{1} ; \quad \sigma(\zeta)=i(1+\zeta) /(1-\zeta), \quad \zeta \in \Delta,
$$

and

$$
W_{t}^{\mu}(\zeta):=W^{\mu}(\zeta, t)=\frac{W^{\mu}(t \zeta)}{W^{\mu}(t)}: \Delta \times \bar{\Delta} \rightarrow \widehat{\mathbb{C}}
$$

then

$$
w_{t}^{\mu}(z):=w^{\mu}(z, t)=\sigma \circ W_{t}^{\mu} \circ \sigma^{-1}(z) .
$$

By the chain rule for the Schwarzians,

$$
S_{w^{\mu}}(\cdot, t)=t^{2} S_{w^{\mu}}(\cdot)=t^{-2}\left(S_{W^{\mu}} \circ \sigma^{-1}\right)\left(\sigma^{\prime}\right)^{-2} .
$$

This point-wise equality determines a family of maps $\eta(\varphi, t)=S_{w_{t}^{\mu}}$ of the space $\mathbf{T}$ into itself, parametrized by $t \in \bar{\Delta}$, with

$$
\eta(\mathbf{0}, t)=\mathbf{0}, \quad \eta(\varphi, 0)=\mathbf{0}, \quad \eta(\varphi, 1)=\varphi .
$$

For any fixed $t$ with $|t|<1$, the function $\eta(\varphi, t)$ is holomorphic in $\varphi$ on $\mathbf{T}$ and by Lemma 5 for any fixed $\varphi$ it is holomorphic in $t$ in the disk $\{|t|<1\}$. In addition, this function is bounded on $\mathbf{T}$ which follows from the estimate

$$
S_{W_{t}^{\mu}}(\zeta)<6|t|^{2} /\left(|\zeta|^{2}-1\right)^{2}, \quad \zeta \in U^{*}
$$

Hence, by Hartogs' theorem extended to complex Banach spaces, the function $\eta(\varphi, t)$ is jointly holomorphic in both variables the function $(\varphi, t) \in \mathbf{T} \times \Delta$.

We apply the homotopy (4) to $\varphi=S_{w^{\mu}} \in \mathbf{T}\left(\Gamma_{0}\right)$. Since it is not compatible with the group $\Gamma_{0}$, there are images $\varphi_{t}:=\eta(\varphi, t)=S_{w_{t}^{\mu}}$ which are located in $\mathbf{T}$ outside of $\mathbf{T}\left(\Gamma_{0}\right)$. The map $\chi \circ \eta(\varphi, t)$ carries these images to the points of the space $\mathbf{T}(0, n)=\mathbf{T}\left(X_{\mathbf{a}^{0}}\right)$. We compose this map with the section $s$ given by Lemma 3 and with a biholomorphism $\xi: s\left(\mathbf{T}\left(X_{\mathbf{a}^{0}}\right)\right) \rightarrow \mathbf{T}\left(\Gamma_{0}\right)$, getting the function

$$
\Theta(\varphi, t)=\xi \circ s \circ \chi \circ \eta(\varphi, t)
$$

which maps holomorphically $\mathbf{T}\left(\Gamma_{0}\right) \times \Delta$ into $\mathbf{T}\left(\Gamma_{0}\right)$ with $\Theta(\varphi, 0)=\mathbf{0}$.

The crucial point of the proof is to establish that the function (5) extends holomorphically to the limit points $(\varphi, 1)$ representing the initial Schwarzians $S_{w^{\mu}}$. This property does not extend (in B-norm) to all points of $\mathbf{T}$.

To prove the limit holomorphy, fix a point $\varphi_{0} \in \mathbf{T}\left(\Gamma_{0}\right)$ and consider in its small neighborhood $V_{0}$ the local coordinates $\mathbf{a}_{*}=\left(a_{1}, \ldots, a_{n-3}\right)$ introduced above.

Both maps $\eta$ and $\Theta$ are holomorphic in the points $\left(\varphi_{0}, t\right)$ of this neighborhood for all $t$ with $|t|<1$. On the other hand, the coordinates $\mathbf{a}_{*}$ are determined by the corresponding quasiconformal maps $w_{t}^{\mu}$ and, together with these maps, are uniformly continuous in $t$ in the closed disk $\{|t| \leq 1\}$. This follows from the uniform boundedness of dilatations given by the estimate

$$
k\left(w_{t}^{\mu}\right)=\left\|\mu_{t}\right\|_{\infty} \leq|t|\|\mu\|_{\infty}<1
$$

(which holds for generic holomorphic motions) and from non-increasing the Kobayashi metric $d_{X}(\cdot, \cdot)$ under holomorphic maps. Since this metric on Teichmüller spaces equals their intrinsic Teichmüller metric $\tau_{\mathbf{T}\left(\Gamma_{0}\right)}$, one gets from (6),

$$
\tau_{\mathbf{T}\left(\Gamma_{0}\right)}(\mathbf{0}, \Theta(\varphi, t))=d_{\mathbf{T}\left(\Gamma_{0}\right)}(\mathbf{0}, \Theta(\varphi, t)) \leq \tanh ^{-1}\left(|t|\|\mu\|_{\infty}\right) .
$$


Hence, the function $\Theta(\varphi, t)$ determines a normal family on $V_{0} \cap \mathbf{T}\left(\Gamma_{0}\right)$.

Applying the classical Weierstrass theorem about the locally uniformly convergent sequences of holomorphic functions in finite dimensional domains, one derives that the limit function

$$
\Theta(\varphi, 1)=\lim _{t \rightarrow 1} \Theta(\varphi, t)
$$

also is holomorphic on $V_{0} \cap \mathbf{T}\left(\Gamma_{0}\right)$, and then on $\mathbf{T}\left(\Gamma_{0}\right)$, which completes the proof of the theorem.

\section{REFERENCES}

[1] L.V. Ahlfors, On quasiconformal mappings, J. Analyse Math. 3 (1954), 1 - 58: correction 207-208.

[2] L.Bers, Spaces of Riemann surfaces, Proc. Internat. Congress Math. 1958, Cambridge Univ. Press, NY, 1960, pp. 349-361.

[3] L. Bers, Fiber space over Teichmüller spaces, Acta Math. 130 (1973), 89-126.

[4] J.G. Clunie, D.J. Hallenbeck and T.H. MacGregor, A peaking and interpolation problem for univalent functions, J. Math. Anal. Appl. 111 (1985), 559-570.

[5] C.J. Earle, On quasiconformal extensions of the Beurling-Ahlfors type, Contribution to Analysis, Academic Press, New York, 1974, pp. 99-105.

[6] C.J. Earle, The holomorphic contractibility of two generalized Teichmüller spaces, Publ. Inst. Math. (Beograd) (N.S.) 75 (89) (2004), 109-117.

[7] C.J. Earle and I. Kra, On sections of some holomorphic families of closed Riemann surfaces, Acta Math. 137 (1976), 49-79.

[8] F.P. Gardiner, Teichmüller Theory an Quadratic Differentials, Wiley, New York, 1987.

[9] E.A. Gorin, Zapiski Nauchn. Seminarov Leningrad. Otdel. Mat. Inst. Steklov. (LOMI) 81 (1978), 58-61 (Problem 9.2); Engl. transl. in J. Soviet Math. 26 (1984), no. 5.

[10] A. Hirchkowitz, A propos de principe d'Oka, C.R. Acad. Sci. Paris 272 (1971), A792-A794.

[11] I. Kaliman, Holomorphic universal covering spaces of polynomials without multiple roots, Funktional Anal. i Prilozh. 71 (1975), no. 1, 71 (Russian).

[12] S. L. Krushkal, Quasiconformal Mappings and Riemann Surfaces, Wiley, New York, 1979.

[13] S.L. Krushkal, On the question of the structure of the universal Teichmüller space, Soviet Math. Dokl. 38 (1989), 435-437.

[14] S.L. Krushkal, Teichmüller spaces are not starlike, Ann. Acad. Sci. Fenn. Ser. A.I. Math. 20 (1995), 167-173.

[15] S.L. Krushkal, Convexity and Teichmüller spaces, Lobachevskii Journal of Mathematics 38, no. 2 (2017), 307-314.

[16] O. Lehto, Univalent Functions and Teichmüller Spaces, Springer-Verlag, New York, 1987.

[17] T.H. MacGregor and D.E. Tepper, Finite boundary interpolation by univalent functions, J. Approx. Theory 52 (1988), 315-321.

[18] S. Nag, The Complex Analytic Theory of Teichmüller Spaces, Wiley, 1988.

[19] M. Toki, On non-starlikeness of Teichmüller spaces, Proc. Japan Acad. Ser. A Math. Sci. 69 (1993), 58-60.

[20] M.G. Zaidenberg, Holomorphic rigidity of polynomial polyhedrons and quasihomogeneity, Global Analysis - Studies and Applcations, Yu.G. Borisovich and Yu.E. Gliklikheds (eds.), Lect. Notes Math. 1453, Berlin, Springer, 1990, pp. 291-307.

[21] M.G. Zaidenberg and V.Ya. Lin, On bounded domains of holomorphy that are not holomorphically contractible, Soviet Math. Dokl. 20 (1979), 1262-1266.

[22] M.G. Zaidenberg and V.Ya. Lin, The finiteness theorems for holomorphic maps, Current problems in mathematics. Fundamental directions, vol. 9. Itogi Nauki i Techniki, Vsesoyuzn. Inst. Nauchn. i Technich. Inform., 1986, pp. 127-193 (Russian). 
Department of Mathematics, Bar-Ilan University, 5290002 Ramat-Gan, Israel

and Department of Mathematics, University of Virginia, Charlottesville, VA 22904-4137, USA 